\title{
105. Operationsstrategie bei gastrointestinalen endokrinen Tumoren
}

\author{
H. D. Becker
}

Klinik für Allgemeinchirurgie der Universität Göttingen, Goßlerstraße 10, D-3400 Göttingen

\section{Surgery in Gastrointestinal Endocrine tumors}

Summary. Endocrine tumors of the gastrointestinal tract are of major clinical interest after the development of highly sensitive specific radioimmunological methods for the determination of hormone plasma concentrations. Several diseases can be attributed to hormone-producing tumors such as organic hyperinsulinism, Zollinger-Ellison-syndrome, Verner-Morrisonsyndrome, glucagonoma-syndrome, etc. The identification of even small tumors by an exact localisation procedure is possible which allows to cure the diseases by enucleation of the tumors. Mainly in gastrinoma patients more extended resection procedures, mainly of the pancreas, are indicated.

Key words: Gastrointestinal hormones - Localisation - Enucleation - Resection.

Zusammenfassung. Endokrine Tumoren des Gastrointestinaltraktes sind durch die Entwicklung hochempfindlicher sensibler radioimmunologischer Meßmethoden zur Bestimmung der Hormonkonzentrationen vermehrt in den Blickpunkt des klinischen Interesses getreten. Verschiedene Symptomenkomplexe wie organischer Hyperinsulinismus, Zollinger-EllisonSyndrom, Verner-Morrison-Syndrom, Glucagonom-Syndrom, etc. konnten Hormon-produzierenden Tumoren zugeordnet werden. Durch eine exakte Lokalisationsdiagnostik ist eine Identifizierung kleiner Tumore möglich, die häufig kurativ enucleiert werden können. Beim Gastrinom dagegen sind häufig ausgedehnte Resektionen vorwiegend des Pankreas notwendig. Schliisselwörter: Gastrointestinale Hormone - Lokalisation - Enucleation - Resektion. 